\title{
Dark Energy and Dark Matter from Emergent Gravity Picture
}

\author{
Hyun Seok Yang ${ }^{1, \star}$ \\ ${ }^{1}$ Center for Quantum Spacetime, Sogang University, Seoul 04107, Korea
}

\begin{abstract}
We suggest that dark energy and dark matter may be a cosmic uroboros of quantum gravity due to the coherent vacuum structure of spacetime. We apply the emergent gravity to a large $N$ matrix model by considering the vacuum in the noncommutative (NC) Coulomb branch satisfying the Heisenberg algebra. We observe that UV fluctuations in the NC Coulomb branch are always paired with IR fluctuations and these UV/IR fluctuations can be extended to macroscopic scales. We show that space-like fluctuations give rise to the repulsive gravitational force while time-like fluctuations generate the attractive gravitational force. When considering the fact that the fluctuations are random in nature and we are living in the (3+1)-dimensional spacetime, the ratio of the repulsive and attractive components will end in $\frac{3}{4}: \frac{1}{4}=75: 25$ and this ratio curiously coincides with the dark composition of our current Universe. If one includes ordinary matters which act as the attractive gravitational force, the emergent gravity may explain the dark sector of our Universe more precisely.
\end{abstract}

\section{Introduction}

Dark energy and dark matter are a great mystery of the 20th century physics, which has not been resolved yet. In retrospect, the resolution of a great puzzle requires the upheaval of a radical new physics. Recall how the blackbody radiation and the photoelectric effect had been resolved at the beginning of the 20th century. We know that these problems could not be solved by simply modifying the Newtonian dynamics and the classical electrodynamics. A radical new paradigm, the so-called quantum mechanics, was necessary to solve these problems. If dark energy and dark matter would be such a case, i.e., the 21 st century version of the blackbody radiation and the photoelectric effect, they would not be understood by simply modifying the general relativity and the quantum field theory. Another novel paradigm, a.k.a. quantum gravity, may be necessary to understand the nature of dark energy and dark matter.

The concept of emergent gravity and spacetime recently activated by the AdS/CFT correspondence advocates that spacetime is not a fundamental entity existed from the beginning but an emergent property from a primal monad such as matrices. The emergent spacetime is a new fundamental paradigm that allows a background-independent formulation of quantum gravity and opens a new perspective to resolve the notorious problems in theoretical physics such as the cosmological constant problem, hierarchy problem, dark energy, and dark matter. Moreover the emergent spacetime picture admits a background-independent description of the inflationary universe which has a sufficiently elegant and

^e-mail: hsyang@ sogang.ac.kr 
explanatory power to defend the integrity of physics against the multiverse hypothesis. We emphasize that noncommutative (NC) spacetime necessarily implies emergent spacetime if spacetime at microscopic scales should be viewed as NC [1]. We will elaborate the emergent gravity from a large $N$ matrix model by considering the vacuum in the NC Coulomb branch satisfying the Heisenberg algebra and argue that dark energy and dark matter may arise as a cosmic uroboros of quantum gravity due to the coherent vacuum structure of spacetime.

\section{Emergent Spacetime and the Dark Side of Universe}

Let us start with a zero-dimensional matrix model with four $N \times N$ Hermitian matrices, $\left\{\phi_{a} \in \mathcal{A}_{N} \mid a=\right.$ $1, \cdots, 4\}$. The equations of motion for the matrix model are given by

$$
\left[\phi_{b},\left[\phi_{a}, \phi_{b}\right]\right]=0,
$$

which must be supplemented with the Jacobi identity

$$
\left[\phi_{a},\left[\phi_{b}, \phi_{c}\right]\right]+\left[\phi_{b},\left[\phi_{c}, \phi_{a}\right]\right]+\left[\phi_{c},\left[\phi_{a}, \phi_{b}\right]\right]=0 .
$$

If we consider the $N \rightarrow \infty$ limit, the large $N$ limit opens a new phase of the Coulomb branch given by

$$
\left.\left[\phi_{a}, \phi_{b}\right]\right|_{\mathrm{vac}}=-i B_{a b} \quad \Rightarrow \quad\left\langle\phi_{a}\right\rangle_{\mathrm{vac}}=p_{a} \equiv B_{a b} y^{b}
$$

where the vacuum moduli $y^{a}$ satisfy the Moyal-Heisenberg algebra $\left[y^{a}, y^{b}\right]=i \theta^{a b}$ with $B_{a b}=\left(\theta^{-1}\right)_{a b}$. This vacuum will be called the NC Coulomb branch. Note that the NC Coulomb branch saves the NC nature of matrices while the conventional commutative vacuum dismisses the property.

Suppose that the fluctuations around the vacuum (3) take the form

$$
\phi_{a}=p_{a}+\widehat{A_{a}}(x, y)
$$

We denote the NC $\star$-algebra on $\mathbb{R}_{\theta}^{4}$ by $\mathcal{A}_{\theta}$ and $\widehat{A}_{a}(y) \in \mathcal{A}_{\theta}$ are four-dimensional NC $U(1)$ gauge fields. The adjoint scalar fields in Eq. (4) now obey the deformed algebra given by

$$
\left[\phi_{a}, \phi_{b}\right]=-i\left(B_{a b}-\widehat{F}_{a b}\right) \in \mathcal{A}_{\theta},
$$

where $\widehat{F}_{a b}=\partial_{a} \widehat{A}_{b}-\partial_{b} \widehat{A_{a}}-i\left[\widehat{A_{a}}, \widehat{A_{b}}\right]$ with the definition $\partial_{a} \equiv \operatorname{ad}_{p_{a}}=-i\left[p_{a}, \cdot\right]$. Note that $\left[\phi_{a},\left[\phi_{b}, \phi_{c}\right]\right]=$ $\widehat{D}_{a} \widehat{F}_{b c}$. Using this result, one can derive the equations of motion and the Bianchi identity of NC $U(1)$ gauge fields from Eqs. (1) and (2), respectively. For these field variables, the Lie algebra homomorphism reads as $\mathcal{A}_{\theta} \rightarrow \mathfrak{D}:\left[\phi_{a},\left[\phi_{b}, \phi_{c}\right]\right] \mapsto\left[\widehat{V}_{a},\left[\widehat{V}_{b}, \widehat{V}_{c}\right]\right]$. In the commutative limit, we thus get the following correspondence $[1,2]$ :

$$
\widehat{D}_{b} \widehat{F}_{a b}=0 \stackrel{|\theta| \rightarrow 0}{\Longrightarrow} \quad\left[V_{b},\left[V_{a}, V_{b}\right]\right]=0, \quad \widehat{D}_{[a} \widehat{F}_{b c]}=0 \quad \stackrel{|\theta| \rightarrow 0}{\Longrightarrow} \quad\left[V_{[a},\left[V_{b}, V_{c]}\right]\right]=0 .
$$

Note that the torsion and curvature are multi-linear differential operators defined by $T(X, Y)=$ $\nabla_{X} Y-\nabla_{Y} X-[X, Y]$ and $R(X, Y) Z=\left[\nabla_{X}, \nabla_{Y}\right] Z-\nabla_{[X, Y]} Z$, where $X, Y$ and $Z$ are vector fields on $M$. Therefore they satisfy the relations $T\left(V_{a}, V_{b}\right)=\lambda^{2} T\left(E_{a}, E_{b}\right)$ and $R\left(V_{a}, V_{b}\right) V_{c}=\lambda^{3} R\left(E_{a}, E_{b}\right) E_{c}$. After imposing the torsion free condition $T\left(E_{a}, E_{b}\right)=0$, it is easy to derive the identity $R\left(E_{[a}, E_{b}\right) E_{c]}=$ $\lambda^{-3} R\left(V_{[a}, V_{b}\right) V_{c]}=\lambda^{-3}\left[V_{[a},\left[V_{b}, V_{c]}\right]\right]$. Consequently we see that the Bianchi identity for NC $U(1)$ gauge fields in the commutative limit is equivalent to the first Bianchi identity for the Riemann curvature tensors, i.e.,

$$
\widehat{D}_{[a} \widehat{F}_{b c]}=0 \stackrel{|\theta| \rightarrow 0}{\Longrightarrow} R\left(E_{[a}, E_{b}\right) E_{c]}=0
$$


The mission for the equations of motion is more involved. But, from the experience on the Bianchi identity (7), we basically expect that it will be reduced to the Einstein equations

$$
\widehat{D}_{b} \widehat{F}_{a b}=0 \stackrel{|\theta| \rightarrow 0}{\Longrightarrow} \quad R_{a b}=8 \pi G\left(T_{a b}-\frac{1}{2} \delta_{a b} T\right) .
$$

After a straightforward but tedious calculation, we get a remarkably simple but cryptic result [2]

$$
R_{a b}=-\frac{1}{\lambda^{2}}\left(g_{d}^{(+) i} g_{d}^{(-) j}\left(\eta_{a c}^{i} \bar{\eta}_{b c}^{j}+\eta_{b c}^{i} \bar{\eta}_{a c}^{j}\right)-g_{c}^{(+) i} g_{d}^{(-) j}\left(\eta_{a c}^{i} \bar{\eta}_{b d}^{j}+\eta_{a c}^{i} \bar{\eta}_{b d}^{j}\right)\right)
$$

To get the above result, we have taken the canonical decomposition of the structure equation as

$$
g_{a b c}=g_{c}^{(+) i} \eta_{a b}^{i}+g_{c}^{(-) i} \bar{\eta}_{a b}^{i}
$$

First it is convenient to decompose the energy-momentum tensor into two parts

$$
\begin{aligned}
& 8 \pi G T_{a b}^{(M)}=-\frac{1}{\lambda^{2}}\left(g_{a c d} g_{b c d}-\frac{1}{4} \delta_{a b} g_{c d e} g_{c d e}\right), \\
& 8 \pi G T_{a b}^{(L)}=\frac{1}{2 \lambda^{2}}\left(\rho_{a} \rho_{b}-\Psi_{a} \Psi_{b}-\frac{1}{2} \delta_{a b}\left(\rho_{c} \rho_{c}-\Psi_{c} \Psi_{c}\right)\right),
\end{aligned}
$$

where $\rho_{a} \equiv g_{b a b}$ and $\Psi_{a}=-\frac{1}{2} \varepsilon^{a b c d} g_{b c d}$. A close inspection reveals that the first one is the Maxwell energy-momentum tensor given by

$$
T_{a b}^{(M)}=\frac{\hbar^{2} c^{2}}{g_{Y M}^{2}}\left(F_{a c} F_{b c}-\frac{1}{4} \delta_{a b} F_{c d} F_{c d}\right)
$$

but the second one seems to be mystic.

The reason is the following. The curvature tensor $R_{a b c d}$ in general relativity can be decomposed according to the canonical split of Lie algebra $s o(4)=s u(2)_{L} \oplus s u(2)_{R}$ and the canonical decomposition of two-forms $\Omega^{2}(M)=\Omega_{+}^{2} \oplus \Omega_{-}^{2}$ as

$$
R=\left(\begin{array}{cc}
W^{+}+\frac{1}{12} s & B \\
B^{T} & W^{-}+\frac{1}{12} s
\end{array}\right) .
$$

A notable point is that the Ricci scalar $s$ appears in the blocks (++) and (--) while the traceless Ricci tensors $B$ and $B^{T}$ show up in the blocks (+-) and (-+). Since the Ricci tensors (9) emergent from NC $U(1)$ gauge fields belong to the mixed sector (+-) and (-+), they should be traceless according the decomposition (14). However the Ricci tensor (9) has a nontrivial Ricci scalar given by $s=$ $\frac{1}{2 \lambda^{2}}\left(\rho_{a} \rho_{a}-\Psi_{a} \Psi_{a}\right)$. Hence the Liouville energy-momentum tensor (12) cannot be realized in the context of general relativity. In order to descry closer aspects of the second energy-momentum tensor, let us separate the scalar and tensor perturbations as $\rho_{a} \rho_{b}=\frac{1}{4} \delta_{a b} \rho_{c} \rho_{c}+\left(\rho_{a} \rho_{b}-\frac{1}{4} \delta_{a b} \rho_{c} \rho_{c}\right)$ and $\Psi_{a} \Psi_{b}=$ $\frac{1}{4} \delta_{a b} \Psi_{c} \Psi_{c}+\left(\Psi_{a} \Psi_{b}-\frac{1}{4} \delta_{a b} \Psi_{c} \Psi_{c}\right)$. In a long wavelength limit where the quadruple modes can be ignored, the Liouville energy-momentum tensor (12) behaves like a cosmological constant [3]

$$
T_{\mu \nu}^{(L)} \approx-\frac{s}{32 \pi G} g_{\mu \nu} .
$$

In order to get a corresponding result in (3+1)-dimensional Lorentzian spacetime, let us take the analytic continuation defined by $x^{4}=i x^{0}$. Under this Wick rotation, $g_{\mu \nu} \rightarrow g_{\mu \nu}, \rho_{\mu} \rightarrow \rho_{\mu}$ and $\Psi_{\mu} \rightarrow i \Psi_{\mu}$, so the Liouville energy-momentum tensor in the Lorentzian signature is given by

$$
T_{\mu \nu}^{(L)}=\frac{1}{16 \pi G \lambda^{2}}\left(\rho_{\mu} \rho_{\nu}+\Psi_{\mu} \Psi_{v}-\frac{1}{2} g_{\mu \nu}\left(\rho_{\lambda}^{2}+\Psi_{\lambda}^{2}\right)\right) .
$$


Note that $\rho_{\mu}$ and $\Psi_{\mu}$ are four-vectors and random fluctuations in nature and the Lorentzian four-vectors have their own causal structure. They are classified into three classes:

(A) $\left(\rho_{\mu}, \Psi_{\mu}\right)$ are space-like vectors, i.e. $g^{\mu v} \rho_{\mu} \rho_{v}>0$, etc.

(B) $\left(\rho_{\mu}, \Psi_{\mu}\right)$ are time-like vectors, i.e. $g^{\mu v} \rho_{\mu} \rho_{v}<0$, etc.

(C) $\left(\rho_{\mu}, \Psi_{\mu}\right)$ are null vectors, i.e. $g^{\mu v} \rho_{\mu} \rho_{v}=0$, etc.

which is in sharp contrast to the Riemannian space where every vectors are positive-definite. We will see that the nature of gravitational interactions depends on their causal structure.

In macroscopic scales where the scalar fluctuations are dominant compared to tensor perturbations, the Raychaudhuri equation can be approximated as

$$
\dot{\Theta} \approx \frac{s}{4}
$$

where $s=\frac{1}{2 \lambda^{2}} g^{\mu \nu}\left(\rho_{\mu} \rho_{v}+\Psi_{\mu} \Psi_{v}\right)$ is the Ricci scalar. Thus the behavior of spacetime expansion/contraction crucially depends on the causal structure of random fluctuations in the classes (A), (B) and (C):

(A) $\dot{\Theta} \approx \frac{s}{4}>0$ when $\left(\rho_{\mu}, \Psi_{\mu}\right)$ are space-like vectors,

(B) $\dot{\Theta} \approx \frac{s}{4}<0$ when $\left(\rho_{\mu}, \Psi_{\mu}\right)$ are time-like vectors,

(C) $\dot{\Theta} \approx \frac{s}{4}=0$ when $\left(\rho_{\mu}, \Psi_{\mu}\right)$ are null vectors.

As a result, space-like fluctuations give rise to the repulsive gravitational force while time-like fluctuations generate the attractive gravitational force. Let us forget about the null case (C) since it does not contribute to the spacetime expansion/contraction.

It should be important to notice that the NC Coulomb branch is generated by the Planck energy condensate in vacuum, i.e., $\rho_{v a c}=\frac{1}{4 g_{Y M}^{2}}\left|B_{\mu \nu}\right|^{2} \sim 10^{-2} M_{P}^{4}[2,3]$. An important point is that the fluctuations over the coherent vacuum (3) should be subject to the spacetime uncertainty relation since the NC Coulomb branch satisfies the Heisenberg algebra. The spacetime uncertainty relation is realized as the UV/IR mixing of vacuum fluctuations. This means that UV fluctuations in the NC Coulomb branch are always paired with IR fluctuations and these UV/IR fluctuations can be extended to macroscopic scales [3]. Hence let us consider the vacuum fluctuations whose IR fluctuations have a macroscopic wavelength $L_{H}=M_{H}^{-1}$. Having this in mind, one can estimate the energy scale of the Liouville energy-momentum tensor (16) by observing that the vacuum fluctuations represented by $\widehat{F}_{\mu v}(y)$ are not localized but extended to a macroscopic scale $L_{H}[2,3]$. Actually the vacuum fluctuation energy

$$
\delta \rho=-\frac{1}{2 g_{Y M}^{2}} B_{\mu \nu} \widehat{F}^{\mu \nu} \sim \frac{1}{L_{P}^{2} L_{H}^{2}}
$$

is the leading contribution to the curvature by the vacuum fluctuations and coming from the boundary term on a hypersurface of radius $L_{H}$. This implies that the Ricci scalar in Eq. (15) has the curvature scale given by $|s| \sim \frac{1}{L_{H}^{2}}$, i.e.,

$$
T_{\mu \nu}^{(L)} \sim \pm \frac{1}{L_{P}^{2} L_{H}^{2}} g_{\mu \nu} .
$$

A few remarks are in order. If the macroscopic scale $L_{H}$ is identified with the size of cosmic horizon of our observable universe, $L_{H}=1.3 \times 10^{26} \mathrm{~m}$, the extended (nonlocal) energy in Eq. (18) or (19) is in good agreement with the observed value of current dark energy $\rho_{D E}:=\delta \rho \approx\left(10^{-3} \mathrm{eV}\right)^{4}$. Moreover one can determine the total energy within the hypersurface of radius $L_{H}$, which is given by $\delta E=\frac{4 \pi L_{H}}{3 L_{P}^{2}}$. Thus the corresponding total entropy $\delta S=\frac{\delta E}{T_{H}}$ is determined as $\delta S=\frac{A_{H}}{4 G}$ since the de Sitter temperature of the cosmological horizon is given by $T_{H}=\frac{1}{2 \pi L_{H}}$, where $A_{H}=4 \pi L_{H}^{2}$ and $8 \pi G=L_{P}^{2}$. Of course the numerical factor is a wishful thinking. This argument shows that the dark 
energy/matter in our Universe would be a holographic manifestation of a microscopic physics, a.k.a. quantum gravity. We showed before that space-like fluctuations give rise to the repulsive gravitational force while time-like fluctuations generate the attractive gravitational force. When considering the fact that the fluctuations are random in nature and we are living in the $(3+1)$-dimensional spacetime, the ratio of the repulsive and attractive components will end in $\frac{3}{4}: \frac{1}{4}=75: 25$ and this ratio curiously coincides with the dark composition of our current Universe [3]. Note that the dark energy in (19) sets the current Hubble parameter $H_{0}=\frac{c}{L_{H}}$ and the Hubble parameter induces a characteristic acceleration scale $a_{0}=c H_{0}=\frac{c^{2}}{L_{H}}$. Since the dark matter is the third of the dark energy, the dark matter will give rise to the attractive acceleration scale $\frac{a_{0}}{3}=\frac{c^{2}}{3 L_{H}}$. This attractive force will compete with ordinary matters depending on their characteristic scales. The inclusion of ordinary matters definitely changes the previous ratio as $75 \downarrow: 25 \uparrow$, that will cause a better match with the current observation. Therefore it is expected that the emergent gravity can explain the dark sector of our Universe more precisely after including ordinary matters in this scheme.

The emergent gravity picture requires to unify geometry and matters on an equal footing. But it is not yet understood what matter is from the emergent gravity picture although a tough idea was suggested in [1-3]. So we simply assume the matter fields that obey the law of Standard Model. Suppose that ordinary matters are added in the background of dark matter and dark energy given by (19). Since the ordinary matter acts as the attractive gravitational force which decays as the $\frac{1}{r^{2}}$ law, two attractive forces due to the dark matter and ordinary matters will compete each other with their own characteristic scales. The gravitational force due to ordinary matters will dominate at small scales while the gravitational acceleration due to dark matter dominates at large scales. To illuminate this aspect, let us consider a (spiral or disk) galaxy and $M(r)$ be the mass contained inside an orbit of radius $r$. The mass distribution of the galaxy gives rise to the acceleration $a_{M}=\frac{G M(r)}{r^{2}}$ which decreases as $1 / r^{2}$ as $r$ increases if there is no mass outside this radius. Thus there is a crossover where the acceleration $a_{M}$ becomes equal to the acceleration $\frac{a_{0}}{3}=\frac{c^{2}}{3 L_{H}}$ due to the dark matter:

$$
a_{M}=\frac{G M}{r^{2}} \lesssim \frac{a_{0}}{3}=\frac{c^{2}}{3 L_{H}} \text {. }
$$

One can see from Eq. (20) that this crossover arises at the distance $r_{c}=\frac{\sqrt{3 G M L_{H}}}{c}$ from the center of the galaxy, over which the acceleration $a_{0} / 3$ due to the dark matter dominates. For example, the crossover distances for M33 $\left(M=5 \times 10^{10} M_{\odot}, R=9 \mathrm{kpc}\right)$ and the Milky Way $\left(M=10^{12} M_{\odot}, R=65 \mathrm{kpc}\right)$ are $r_{c} \approx 5.6 \mathrm{kpc}\left(1.7 \times 10^{20} \mathrm{~m}\right)$ and $29.8 \mathrm{kpc}\left(9.2 \times 10^{20} \mathrm{~m}\right)$, respectively, which are roughly half the size of the galaxies. This implies that the flattening of the galaxy rotation curve may be explained by the dark matter given by Eq. (19).

\section{Acknowledgments}

This work was supported by the National Research Foundation of Korea (NRF) grant funded by the Korea government (MOE) (No. NRF-2015R1D1A1A01059710).

\section{References}

[1] H. S. Yang, Int. J. Mod. Phys. A 30, 1550016 (2015).

[2] H. S. Yang, J. High Energy Phys. 05, 012 (2009).

[3] H. S. Yang, J. Phys. Conf. Ser. 343, 012132 (2012). 